\title{
Ultracold Neutron Source at the PULSTAR Reactor: Engineering Design and Cryogenic Testing
}

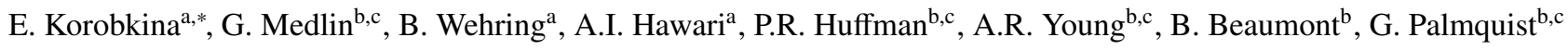 \\ ${ }^{a}$ Department of Nuclear Engineering,North Carolina State University, 2500 Stinson Drive, Box 7909, Raleigh, NC 27695, USA \\ ${ }^{b}$ Department of Physics, North Carolina State University, 2401 Stinson Drive, Box 8202, Raleigh, NC 27695, USA \\ ${ }^{c}$ Triangle Universities Nuclear Laboratory, 116 Science Drive, Box 90308, Durham, NC 27708
}

\begin{abstract}
Construction is completed and commissioning is in progress for an ultracold neutron (UCN) source at the PULSTAR reactor on the campus of North Carolina State University. The source utilizes two stages of neutron moderation, one in heavy water at room temperature and the other in solid methane at $\sim 40 \mathrm{~K}$, followed by a converter stage, solid deuterium at $5 \mathrm{~K}$, that allows a single down scattering of cold neutrons to provide UCN. The UCN source rolls into the thermal column enclosure of the PULSTAR reactor, where neutrons will be delivered from a bare face of the reactor core by streaming through a graphite-lined assembly. The source infrastructure, i.e., graphite-lined assembly, heavy-water system, gas handling system, and helium liquefier cooling system, has been tested and all systems operate as predicted. The research program being considered for the PULSTAR UCN source includes the physics of UCN production, fundamental particle physics, and material surface studies of nanolayers containing hydrogen. In the present paper we report details of the engineering and cryogenic design of the facility as well as results of critical commissioning tests without neutrons.
\end{abstract}

Keywords: ultracold neutrons, moderation, nuclear reactor, ortho-para conversion, raman spectroscopy

\section{Introduction}

2 sembly.
The design of the ultracold neutron (UCN) source $[1,2]$ at the PULSTAR nuclear reactor on the campus of North Carolina State University has a cryostat placed in a tank of heavy water located in the reactor thermal column after all of the graphite has been removed. The cryostat contains $200 \mathrm{~g}$ of solid deuterium $(4.5 \mathrm{~cm} \times 17 \mathrm{~cm}$ diameter $)$ at a temperature of $5 \mathrm{~K}$ surrounded by $600 \mathrm{~g}$ of solid methane $\left(1 \mathrm{~cm}\right.$ thick cup-shaped cold ${ }^{22}$ source) at a temperature of $\sim 40 \mathrm{~K}$. Fast and thermal neutrons leaving a bare face of the reactor core stream into the heavy- ${ }^{24}$ water source tank through a large void in a graphite-lined as- ${ }^{25}$

The PULSTAR nuclear reactor is a $1 \mathrm{MW}$ open tank reactor that is light water moderated and cooled. Detailed calcula-

*Corresponding author. Tel.: (919) 515-3302; Fax: (919) 513-1276;

Email address: ekorobk@ncsu .edu (E. Korobkina)
15 17

18
20 tions are being completed in support of a license amendment to upgrade the PULSTAR power to $2 \mathrm{MW}$ [3]. The PULSTAR is fueled with sintered pellets of $\mathrm{UO}_{2}$ enriched to $4 \%$, similar to power reactor fuel. This design requires significantly more U-235 than other $1 \mathrm{MW}$ research reactors, viz., TRIGA, and the use of replaceable neutron reflectors (water, graphite, and beryllium). The advantages of the PULSTAR design are longer fuel lifetimes and high neutron leakage from a bare reactor face, both benefiting our installation of a UCN source.

UCN are neutrons with energies less then a few hundred neV. Due to their low energy, they are reflected from certain material surfaces at any angle of incidence and can thus be confined in containers for a few thousand seconds. Additionally, magnetic and gravitational potentials are of similar energy scales. A combination of any of the three can be used in UCN confinement.

UCN are of special interest in high precision measurements 


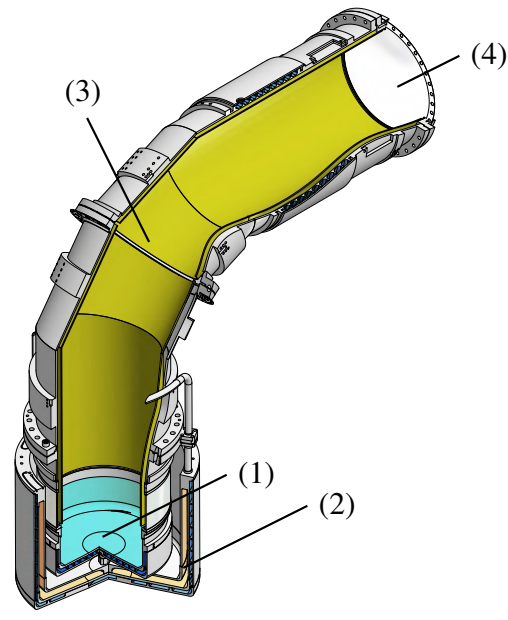

(a)

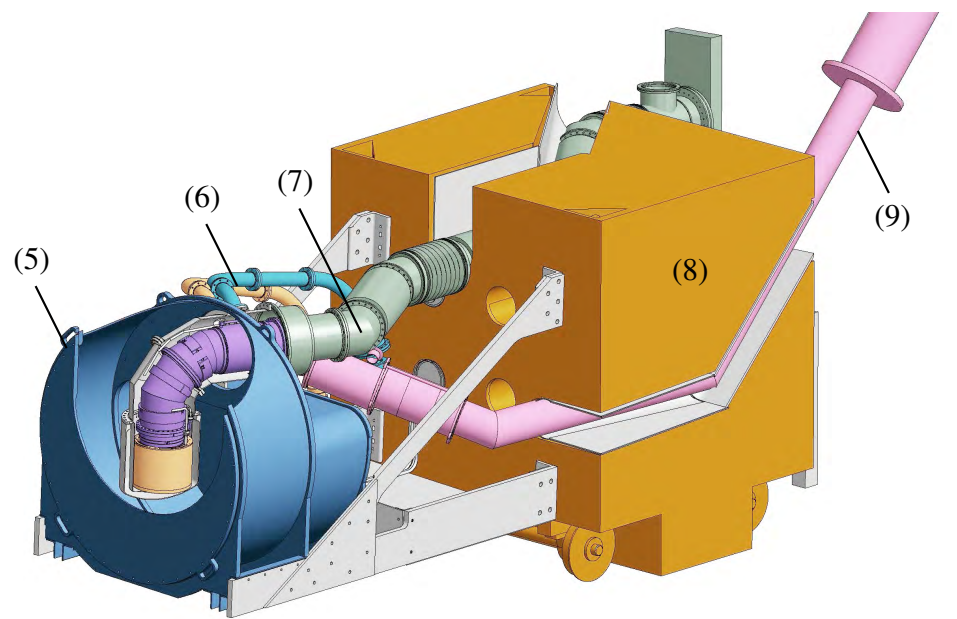

(b)

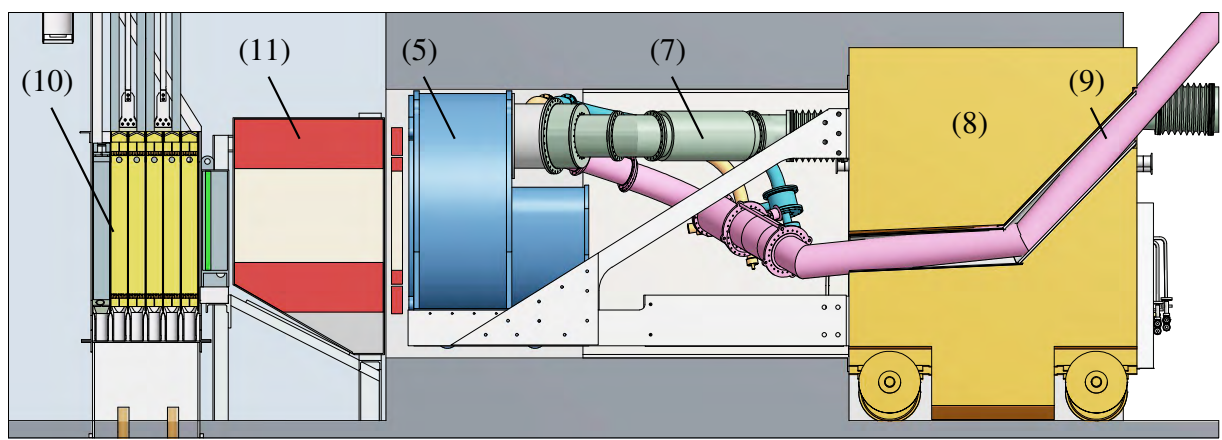

(c)

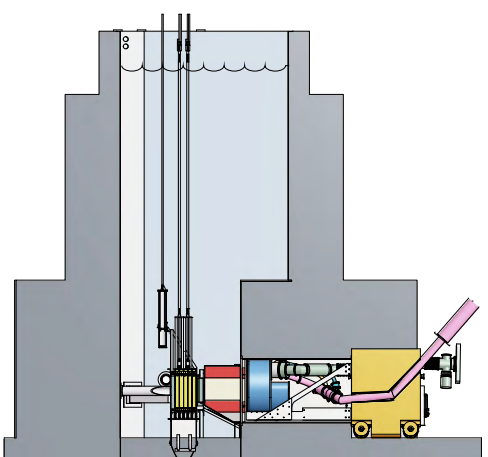

(d)

Figure 1: A cutaway engineering drawing of the UCN source cryostat, as envisioned installed in the reactor thermal column, is shown in (a), including the (1) deuterium container, (2) methane container, (3) UCN guide, and (4) UCN window foil. The UCN source assembly mounted on the shielding door is shown in (b), including the (5) heavy-water tank, (6) deuterium and methane gas inlets, (7) UCN guide, (8) thermal column shield door, and (9) liquid helium transfer line. The heavy-water tank and cryostat vacuum jacket have been cut away to show the cryostat. A cross section of the reactor showing the source assembly in the thermal column is shown in (c) and (d), where (10) is the reactor core and (11) is the neutron transport system. In all drawings, neutrons from the reactor core enter from the left and UCN exit to the right of the shielding door.

in fundamental particle physics [4]. For example, measurement 42 of the neutron electric-dipole moment (nEDM) is recognized as 43 one of the most effective probes for physics beyond the Stan- 44 dard Model of particle physics, and has emerged as one of the 45 highest priority measurements for the fundamental symmetries 46 community $[5,6]$. Other experiments include measurements ${ }_{47}$ of the neutron lifetime $[7,8]$ and angular correlations in free ${ }_{48}$ neutron decay [9]. The need to improve the statistical accu- 49 racy of experiments has motivated the construction of $\mathrm{UCN}_{50}$ sources around the world. New facilities rely mostly on the ${ }_{51}$ spallation reaction as a primary source of neutrons [10-12]. In ${ }_{52}$ addition smaller university reactors have became new hosts of UCN source projects. These small reactor facilities can provide test beds for larger scale experiments, as well as provide students on-campus access to world class UCN research techniques in smaller scale experiments.

With optimized superthermal source design, the universitybased reactor facilities can be competitive for a number of experiments [13]. The UCN source at the pulsed TRIGA reactor in Mainz has recently demonstrated UCN densities of $10 \mathrm{~cm}^{-3}$ in a 10 liter storage bottle, with a projected pulsed density of $50 \mathrm{~cm}^{-3}$ after future improvements [14]. This is promising for 
storage experiments that are filled periodically.

The source at North Carolina State University will be oper- 90 ated in a steady-state mode. It is anticipated to have a UCN 91 density of $30 \mathrm{~cm}^{-3}$, which allows both continuous and storage 92 experiments, and provides a higher time-averaged density. $\mathrm{A}^{93}$ cryogenic apparatus with a liquid-helium-filled UCN storage ${ }^{94}$ cell, that will be used to test some aspects of the SNS neu- 95 tron electric dipole moment project prior to construction of the 96 main edm apparatus, has been designed to utilize the PULSTAR 97 UCN source. Our team is also working on material science ap- 98 plications that will benefit from the continuous UCN supply.

\section{Engineering Design of the PULSTAR}

\section{UCN Source Assembly}

The conceptual design of the PULSTAR UCN source can $_{104}$ be found in [2]. Here, we briefly review the primary compo- ${ }_{105}$ nents of the design. The overall goal is to optimize the num- ${ }_{106}$ ber of UCN available for experiments. This is accomplished ${ }_{107}$ by decelerating neutrons in three stages - fast to thermal, ther- ${ }_{108}$ mal to cold, and cold to ultracold. The moderator materials are ${ }_{109}$ heavy water at $T \sim 300 \mathrm{~K}$ to provide thermal moderation, solid $_{110}$ methane at $T \sim 40 \mathrm{~K}$ for cold moderation, and solid deuterium at $T=5 \mathrm{~K}$ serves as the ultracold neutron converter. To distinguish it from a moderator, a "converter" is the material in $\mathrm{UCN}_{113}$ sources that downscatters neutrons in single collisions [15]. ${ }_{114}$ The optimized geometry was determined using MCNP simula- ${ }_{115}$ tions of the neutron fluence, while ANSYS-CFX thermal flow simulations were used for optimization of the cryogenic design. The 3D engineering model of the facility is shown in Figure 1 along with a cross-sectional view of the cryostat insert. ${ }^{117}$ The insert contains two separate cryogen containers. The out- ${ }_{118}$ side cup-shaped shell contains the methane, and the inner elbow ${ }_{119}$ houses the neutron guide elements and the solid deuterium.

120

The deuterium container has two heat exchangers to maintain ${ }_{121}$ a difference in temperature between the bottom where $\mathrm{UCN}$ are ${ }_{122}$ produced in the deuterium crystal and the neutron guide where ${ }_{123}$ UCN are extracted from the cryostat. The higher temperature of ${ }_{124}$
9 the guide is needed to prevent deuterium condensation, which would cause additional UCN losses. A welded insert constructed from zircalloy explosively bonded to aluminum provides the thermal separation between the deuterium and guide temperature regions. The deuterium and methane are stored as gas in room temperature tanks and are condensed into the cryostat using the gas handling system described in Section 3.2.

The vacuum-insulated cryostat elbow is installed in a tank filled with 570 liters ( 150 gallons) of heavy water. The entire assembly (shown in Figure 1) is positioned on a support frame attached to the movable heavy-concrete thermal column door. This geometry allows easy removal of the entire source from the thermal column for service. The graphite-lined assembly for neutron transport between the bare face of the core and the $\mathrm{D}_{2} \mathrm{O}$ thermal moderator tank is described in greater detail in Section 3.4.

An on-site helium liquefier provides continuous cooling of the cryostat. Our design features three parallel cooling loops, one for each of the cryostat heat exchangers. Parallel flow allows more flexible and independent temperature control (unlike in-series flow at other UCN facilities). The cyogenic system is described in more detail in Section 3.1

At present, all infrastructure components of the source, including the heavy-water system, gas handling systems, helium liquefier cooling system, graphite-lined assembly, and the source cryostat have been manufactured and assembled for commissioning tests.

\section{Commissioning Tests}

\subsection{Cryogenic System}

The schematic layout of the helium cryogenic system is shown in Figure 2. A Linde model 1430S liquefier supplies liquid helium to a 500 liter helium storage dewar used as a buffer volume. The storage dewar allows one to independently maintain cryostat cooling for several hours in the event of power loss or necessary liquefier maintenance. Helium from the storage dewar enters a custom transfer tube connected to three paral- 


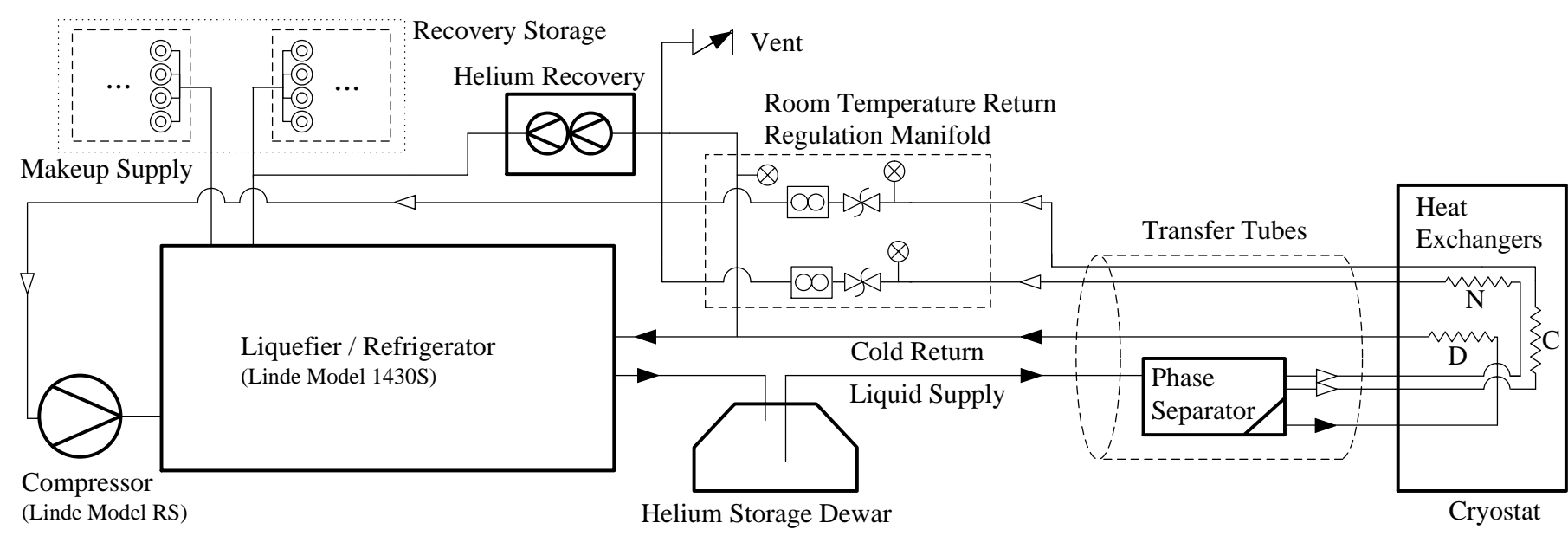

Figure 2: Schematic of the helium cryogenic system where the labels D, N, and C in the cryostat designate the deuterium container, neutron guide, and methane heat exchangers respectively.

lel cooling loops, labeled D-, C-, and N-loops corresponding to 150 the deuterium, methane $\left(\mathrm{CH}_{4}\right)$, and neutron guide cryostat heat ${ }_{151}$ exchangers. The transfer tube includes a built-in phase separa-152 tor that gravitationally separates the liquid phase from the gas ${ }_{153}$ phase. The liquid phase is delivered through the D-loop to cool ${ }_{154}$ the deuterium container, while the pure gas phase is delivered ${ }_{155}$ through the C-loop to the methane container. Depending on ${ }_{156}$ the level in the phase separator, a liquid/gas mixture is deliv- ${ }_{157}$ ered through the N-loop to the neutron guide. Each heat $\mathrm{ex}^{-}{ }_{158}$ changer is paired with temperature sensors and electric heaters. ${ }_{159}$ After exiting the cryostat, return gas from the C- and N-loops ${ }_{160}$ are used in a counter-flow mode to actively shield the supply ${ }_{161}$ lines, minimizing radiative heat loads in the transfer tube. The ${ }_{162}$ exiting gas is then warmed in the room temperature return man- ${ }_{163}$ ifold before returning to the liquefier compressor through two $_{164}$ room temperature proportional valves. The cold gas from the ${ }_{165}$ D-loop is returned directly to the liquefier cold box through ${ }_{166}$ a low-temperature bayonet and cryogenic proportional valve. ${ }_{167}$ When the system is warmed up, pure helium is collected back ${ }_{168}$ into the high pressure recovery storage using a two step recovery system depicted in Figure 2.

Previously the helium liquefier was commissioned with only ${ }_{171}$ the storage dewar. The primary goal of the present testing was ${ }_{172}$ to characterize the cooling power of the cooling loops inside ${ }_{173}$ the cryostat, as well as customize automatic operation of the liquefier. For maximum safety as well as accessibility, the initial cryogenic testing was performed with the source cryostat positioned outside of the reactor biological shield without the room temperature UCN guide (see Figure $3 b$ ). In addition, nonflammable gasses were used to characterize the cryogenic components prior to introducing methane and deuterium.

To begin the testing, 200 liters of the liquid helium inventory were liquefied into the storage dewar. The liquefier was first operated at full power to both liquefy helium into the storage dewar and cool the cryostat. It took less than two days to reach minimum operating temperatures. Typical base temperatures were $5.5 \mathrm{~K}, 12 \mathrm{~K}$, and $35 \mathrm{~K}$ for the deuterium, methane, and neutron guide heat exchangers respectively. Once the cryostat reached base temperatures and the helium inventory had been condensed, the liquefier power was reduced, allowing the system to operate in steady-state mode, maintaining the net liquid helium inventory.

Heater power versus equilibrium temperature was then characterized for each cooling loop for several helium flow rates. During the measurements, typical return gas flow rates were 10 liters/min of gas at STP for both the N- and C-loops. (We are unable to measure the cold D-loop return flow).

The system was able to maintain the desired temperatures in the cryogenic volumes with applied electric heater power well 


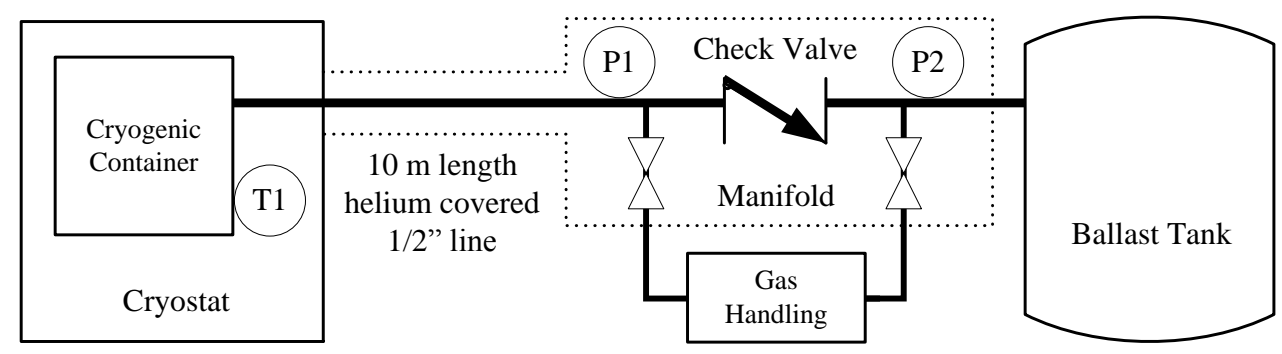

(a)

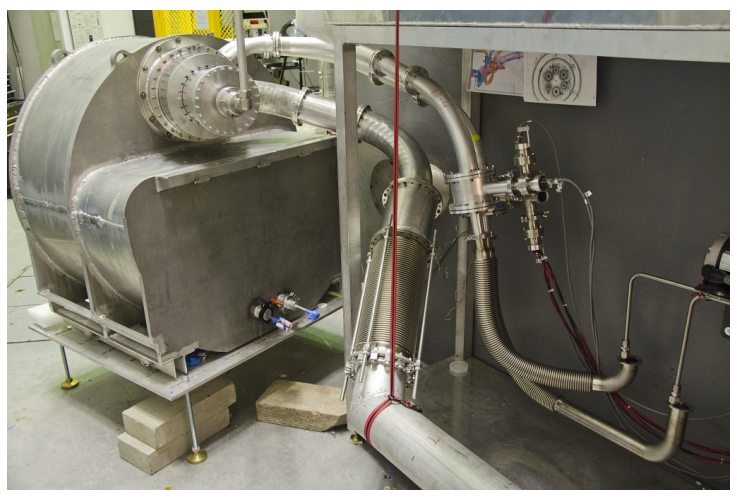

(b)

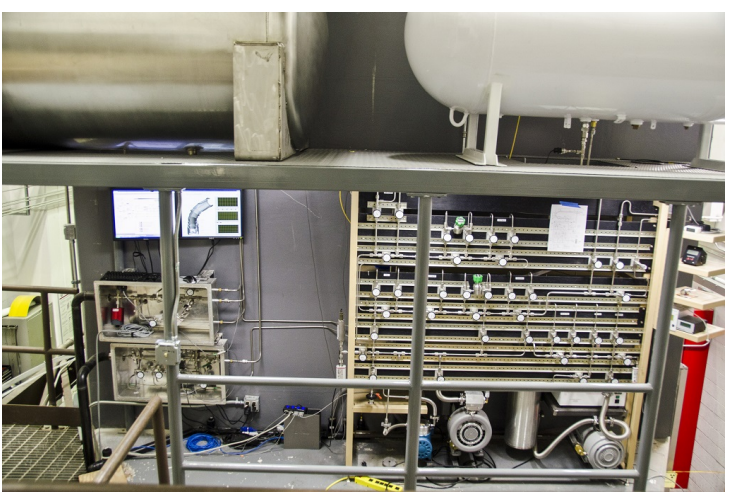

(c)

Figure 3: Photos of the completed (b) source assembly and (c) gas handling systems. Both methane and deuterium systems follow the same basic design shown in the schematic in (a). In (b), the gas lines and helium transfer line are shown entering from the right. In (c), the gas handling systems panel is on the right, the manifolds are inside the aluminium boxes on the left, and the ballast tanks can be seen at the top.

above the expected reactor heat load. The methane container ${ }_{183}$ requires the largest range of temperature control, so a subset of ${ }_{184}$ the heater power and helium flow versus temperature is shown ${ }_{185}$ in Table 1. A heater power of up to $10 \mathrm{~W}$ did not affect the ${ }_{186}$ temperature of the deuterium container.

\begin{tabular}{c|lll} 
Helium Flow & \multicolumn{3}{|c}{ Heater Power $(\mathrm{W})$} \\
$(\mathrm{l} / \mathrm{min})$ & 0 & 10 & 25 \\
\hline 34 & $10 \mathrm{~K}$ & $25 \mathrm{~K}$ & $35 \mathrm{~K}$ \\
27 & $10 \mathrm{~K}$ & $30 \mathrm{~K}$ & $40 \mathrm{~K}$ \\
15 & $10 \mathrm{~K}$ & $30 \mathrm{~K}$ & $60 \mathrm{~K}$ \\
10 & $15 \mathrm{~K}$ & $40 \mathrm{~K}$ & $80 \mathrm{~K}$
\end{tabular}

Table 1: Equilibrium temperature of the methane cryocontainer with electric heater input and helium flow, measured as room temperature return gas.

These tests also showed that our parallel cryogenic loops al-197 low independent temperature control of all three heat exchang-198 ers over a wide range of helium flows without disturbing the 199 stability of the liquefier operation.

\subsection{Gas Handling Systems}

The design of the gas handling systems for methane and deuterium both follow the same logic, shown in Figure 3a. Each system has a ballast tank that is used to measure out a welldefined gas inventory given by the pressure "P2" in Figure 3a lium.

The pressure gauge "P1" measuring pressure above the cryo200 genic containers is located about $10 \mathrm{~m}$ away from the cryogenic 


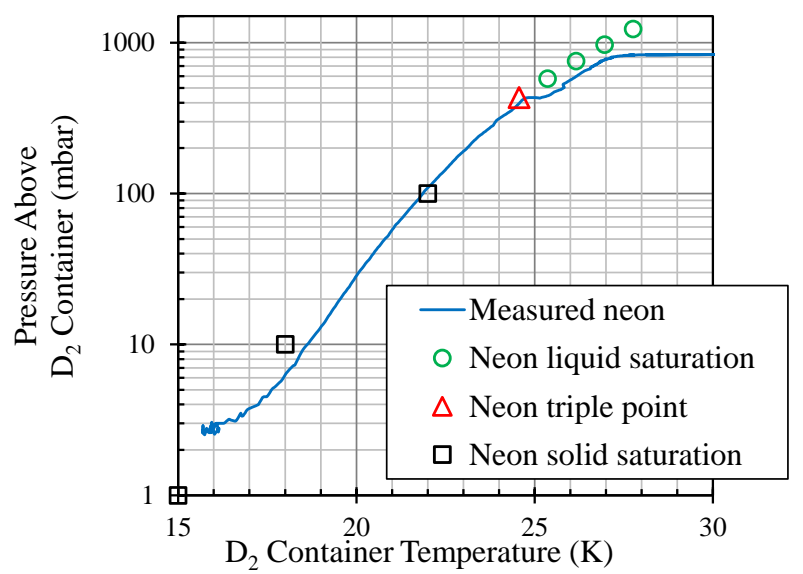

(a)

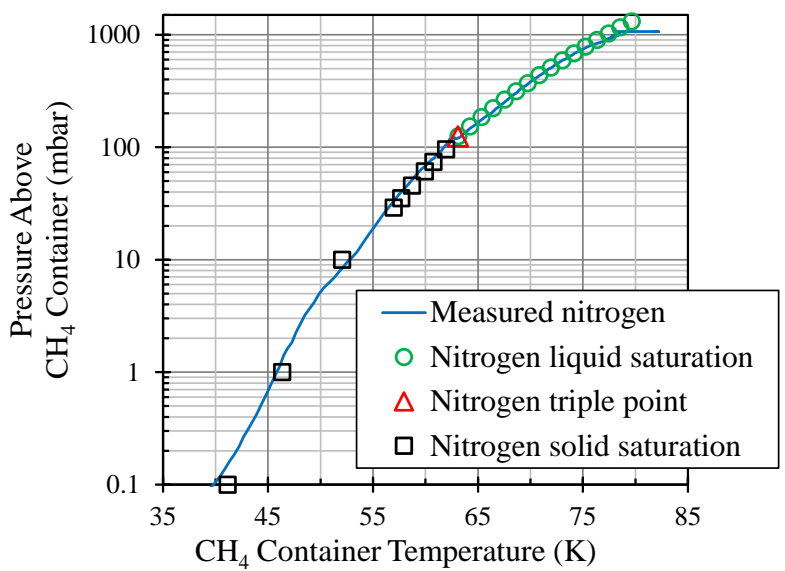

(b)

Figure 4: Measured pressures and measured temperatures of the cryogens, compared with their saturation pressures, while condensing (a) neon and (b) nitrogen in place of deuterium and methane, respectively. This shows the condensing moderators are near equilibrium over a wide range of pressures. The lowest pressures shown in the $\mathrm{D}_{2}$ container are at the minimum of the high-range pressure gauge.

volumes at room temperature. While condensing, and later 224 warming, "P1" and "T1" should show good agreement with the 225 saturation vapor pressure. As a safety precaution, a return check $\mathrm{k}_{226}$ valve on each gas system provides a passive relief path back to $_{227}$ the ballast tank, permitting vapor from warming crystals to ex- ${ }_{228}$ pand back to their ballast tanks at any time.

As an additional safety precaution, all tubing and valves di-230 rectly connected to the cryostat are covered in a helium-filled ${ }_{231}$ jacket. This allows for easy identification of any possible leak $_{232}$ while preventing cryopumping of air through a possible leak ${ }_{233}$ into the cryogenic volumes.

Great care was taken in maintaining the cleanliness of the $e_{235}$ systems. Both gas handling systems are made entirely from instrumentation grade stainless tubing and almost exclusively ${ }_{236}$ with orbital welds and Swagelok VCR metal gasket seals. This is particularly important for the deuterium system, where the $\mathrm{e}^{237}$ purity of the deuterium over a long operational time is required ${ }^{238}$ to maintain performance.

The methane and deuterium gas handling systems were successfully used to condense nonflammable gases into the cryo- ${ }^{24}$ stat, nitrogen in place of methane and neon in place of deu- ${ }^{242}$ terium. Both were first liquefied, then allowed to freeze while ${ }^{243}$ valved off from their ballast tanks. After some period at min- imum temperature, the gases were allowed to warm passively, demonstrating the safe design of the system.

Data for gases in both containers showed excellent agreement with their saturation curves as well as easily identifiable phase transitions (see Figure 4). This shows that gas above the cryogenic container is near equilibrium, an assumption necessary for modeling condensation, and that the pressure measured away from the container is an accurate representation.

In future source operations, methane will be condensed in the above manner. However, the deuterium system is designed to allow gas to grow a deuterium crystal from a vapor phase at lower pressure, and this mode will be used in a later test.

\subsection{Deuterium Para-to-Ortho Conversion}

One crucial part of successful UCN production is limiting the concentration of para-deuterium [16]. The rotational state of deuterium's symmetric molecule couples with its nuclear spin, preventing a change in rotation state number from odd total nuclear spin (para) to even total nuclear spin (ortho) or vice versa. The para species has the more energetic ground state, because it cannot lose energy below the first rotational state, and so can upscatter UCN via a spin flip to the ortho state. This process dramatically reduces UCN yield. The equilibrium para- 


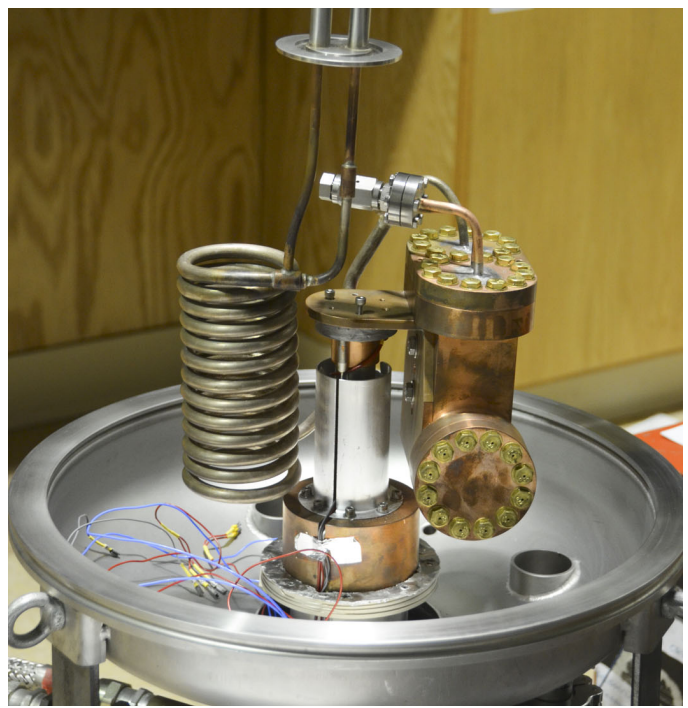

Figure 5: The deuterium spin converter with the vacuum jacket removed, showing the catalyst cell and tube-in-tube heat exchanger.

deuterium concentration is reduced at low temperatures, sufficient for UCN production, but takes many days to convert from the 2-to-1 room temperature ortho to para ratio [17]. Although a radiation field facilitates this process, it will still be too slow to be a practical solution at our source due to the small radiation flux. Fortunately, this process can be accelerated in a magnetic environment that allows for spin flips.

Based on the previous work of C.-Y. Liu and others [1821], systems were developed to both prepare deuterium with a very high ortho- fraction: a para-to-ortho converter and a Raman spectrometer optimized to monitor the para-deuterium and hydrogen content of the source.

Our converter, pictured in Figure 5 consists of a U-shaped copper cell with inlet and outlet gas lines, filled with a catalyst, and connected to the cold head of a cryopump. The converter can be used in two different modes both of which are being evaluated. Deuterium can be condensed and then evaporated in a batch process, or flowed continuously while operating at the triple point of deuterium.

The converter utilizes the paramagnetism of a catalyst material to induce spin flips. Both chromium(VI) trioxide and iron(III) hydroxide were tested as catalysts and showed comparable para-ortho fractions. Chromium trioxide is commer-

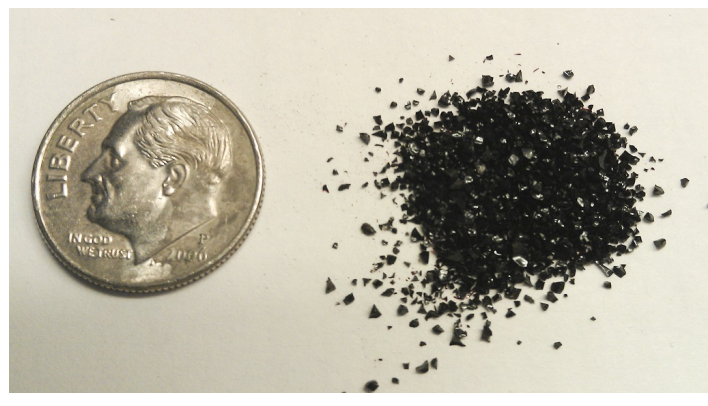

Figure 6: Granules of iron hydroxide.

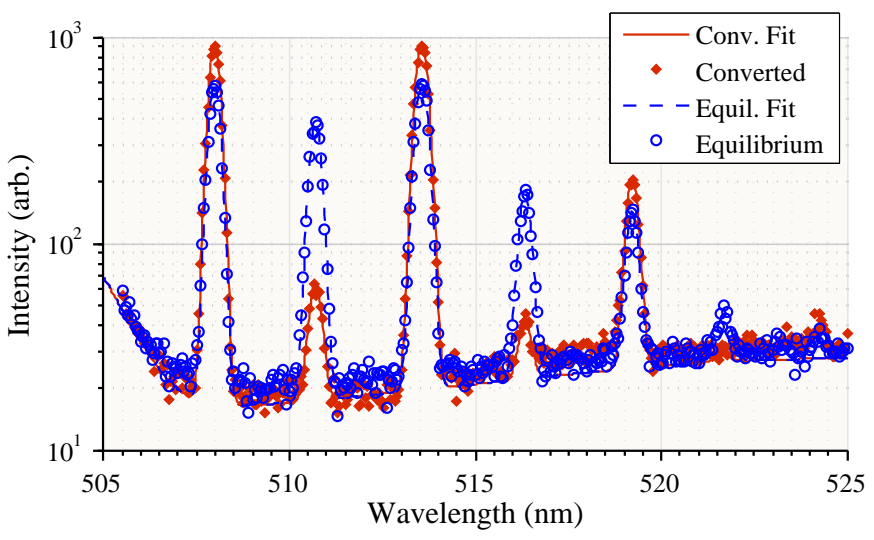

Figure 7: First seven Stokes Raman lines of deuterium, when comparing a room temperature reference (circles) with a spin converted sample (diamonds), gives a para/ortho ratio of less than $4 \%$ following [22].

cially available as Oxisorb (manufactured by Messer Griesheim $\mathrm{GmbH}$, Germany, and available locally through GTS-Welco). Iron hydroxide was made in house by reacting ammonium hydroxide and iron chloride ${ }^{1}$, then drying in a manner that produces small granules, see Figure 6. These granules are small enough to provide a large surface area while also hopefully large enough to prevent small particles of catalyst material from migrating out of the converter volume. Iron hydroxide is appealing because of the toxicity of chromium trioxide, although both materials are being evaluated.

As Liu et al. demonstrated, Raman spectroscopy can be used to precisely determine the para to ortho ratio of the source deuterium, and is also sensitive to hydrogen and HD contaminants. Hydrogen-containing species are a source of UCN loss, and must be maintained at or below about the $0.2 \%$ concentration level to avoid seriously degrading the source performance.

\footnotetext{
${ }^{1}$ We obtained our recipe for iron hydroxide from Steve Lamoreaux.
} 
The converter was tested independently of the gas handling g $_{322}$ system in the condensed batch process mode and yielded a paraortho fraction of a few percent. The catalyst absorbs a great deal ${ }_{324}$ of gas at low temperature, lowering the vapor pressure well be- -325 low saturation pressure. The cell had to be warmed until gas 326 could be sampled at near an atmosphere to improve the sensi-з27 tivity of the Raman spectroscopy analysis.

In one such test, deuterium was introduced over the iron hy-329 droxide catalyst, allowed to sit for 15 minutes at $19 \mathrm{~K}$, then ${ }^{330}$ warmed to $28 \mathrm{~K}$ to be sampled at atmospheric pressure. By ${ }^{331}$ comparison with a room temperature standard, the Raman spec- 332 trum of the sample, pictured in Figure 7, shows 4\% para-ззз deuterium content.

In the final setup, gas will be sampled at a low pressure and ${ }^{335}$ compressed to avoid warming up the converter volume. We alsoзз6 hope that operating in the flow through mode at the triple point ${ }_{337}$ will further lower the ortho to para ratio.

\subsection{Graphite-Lined Neutron-Transport Assembly}

A unique feature of our design is that the source is placed in $_{341}$ the thermal column enclosure of the PULSTAR reactor facility, ${ }_{342}$ where fast and thermal neutrons are delivered from the core $b_{343}$ streaming through a graphite-lined neutron-transport assembly. While the original graphite column provided a well moderated flux, this transport system was designed to take advantage of the high fast neutron leakage of the PULSTAR core and guide both fast and thermal neutrons to the source heavy-water tank with minimum losses. The heavy-water tank of the source serves to thermalize epithermal and fast neutrons.

Originally, the reactor's thermal column consisted of a square column of graphite running through the reactor biological ${ }_{350}$ shield from the outside of the reactor tank liner. A graphite 351 "nosepiece" displaced the water between the reactor tank liner 352 and the core. This was intended to provide a pure thermal neu- ${ }^{-}$ tron flux by thermalization in the graphite in the nosepiece and $d_{354}$ thermal column.

For the UCN source, the graphite nosepiece was replaced 356 with a removable graphite lined assembly called the "nose 357 port." It features a water floodable $45 \mathrm{~cm} \times 45 \mathrm{~cm} \times 70 \mathrm{~cm}$ central void, surrounded on the perimeter by $20 \mathrm{~cm}$ of graphite in an aluminium box. This allows a high neutron flux in the thermal column, acting as a collimator. The nose port can also act as a neutron shutter; when neutrons are required for the source, water can be forced out of the void upon pressurizing with helium gas. A "shielding box" that contains $2.5 \mathrm{~cm}$ (1 inch) of lead is placed between the core and the entrance of the nose port to reduce heating of the UCN source by core gammas. This box can be removed independent of the nose port to change the type or thickness of the shielding material.

Operation of the nose port and shielding box, displacing water with helium, was demonstrated, and the heavy water circulation system was used to fill and empty the moderator tank.

To confirm the performance of the heavy-water thermal moderator tank and verify the expected thermal neutron flux to the source, a mock-up of the heavy-water source tank was constructed. This aluminum "test tank" consisted of a horizontal cylindrical tank the same size as the source tank, placed in the future location of the source heavy-water tank, see Figure 1c. Thermal neutron fluxes were measured down the length of the tank with the gold foil activation method.

The measured fluxes agree well with a MCNP6 [23] simulation, and can be used to benchmark the MCNP model. This will allow prediction of the flux to the UCN converter and suggest possible improvements. A manuscript is being prepared giving detailed analysis of neutron transport to the UCN source.

\section{Summary \& Outlook}

Construction of the source assembly is complete. The cryostat has been successfully cooled to operating temperatures with the helium liquefier system, and we have demonstrated with an artificial heat load simulating reactor radiation heating that operational temperatures can be maintained. The system has been used to condense nonflammable gases in both the deuterium and methane containers.

Preparation for tests with deuterium and methane are 
presently underway. We plan to conduct a detailed study of f $_{392}$ deuterium solidification in our cryostat before installation into the thermal column. The cryostat is currently being modified to allow visual monitoring of the deuterium crystal, fine measurement of the vapor pressure, and embedding of temperature ${ }_{394}$ sensors in the crystal. The spin converter will be tested as part ${ }^{395}$ of the gas handling system tests. Finally the assembly will be ${ }^{396}$ moved into the thermal column for additional tests with neutrons.

A unique feature of our cryogenic design is that the methane $e^{400}$ moderator temperature can be varied as needed; the final oper- ${ }^{401}$ ational temperature is linked to the physics of $\mathrm{UCN}$ production. ${ }_{403}$ Since the cold neutrons are down-scattered in individual colli-404 sions with a single lattice phonon in the solid deuterium, the $\mathrm{e}^{405}$ maximum UCN yield will depend heavily on the intensity of ${ }_{407}^{406}$ the cold neutrons with well-defined energies corresponding to $_{408}$ the maxima in the deuterium density of states. While the $\mathrm{UCN}^{409}$ production cross section versus cold neutron energy has been ${ }^{410}$ measured in two experiments [24, 25], agreement with theoretical predictions is only at the $50 \%$ level. Therefore we plan to ${ }_{413}$ vary the methane temperature to optimize UCN production as ${ }^{414}$ one of the first tests of the source with neutrons.

\section{Acknowledgements}

Authors would like to express their gratitude to PULSTAR ${ }^{423}$ rector staff, especially Andrew Cook, the NCSU College of Sci- ${ }^{424}$ ences machine shop for their high level and friendly technical support, as well as Jonathan Coburn, Camen Royse, and Aaron ${ }_{427}$ Thomas for their help with assembly. Manufacturing of the ${ }^{428}$ cryostat would be impossible without unique welding knowl- ${ }^{429}$

This work was supported in part by the U.S. Department ${ }^{432}$ of Energy under grant number DE-FG02-97ER41042 and by ${ }^{433}$ the National Science Foundation under grant number $\mathrm{PHY}_{435}$ 1307426 .

\section{References}

\section{References}

[1] B. Wehring, A. Young, Trans. Am. Nucl. Soc. 83 (2001) 120.

[2] E. Korobkina, et al., Nucl. Instr. Meth. A 579 (1) (2007) 530-533. doi : 10.1016/j.nima.2007.04.116.

[3] A. Hawari, 2013, IGORR Conference, Daejeon, South Korea 2013. [link]. URL www . igorr.com

[4] F. Wietfeldt, G. Greene, Rev. Mod. Phys. 83 (4) (2011) 1173-1192. doi : 10.1103/RevModPhys.83.1173.

[5] C. Baker, et al., Phys. Rev. Letters 97 (13) (2006) 131801-131804. doi : 10.1103/PhysRevLett.97.131801.

[6] S. Lamoreaux, R. Golub, J. Phys. G: Nucl. Part. Phys. 36 (2009) 104002.

[7] S. Arzumanov, et al., Phys. Letters B 483 (1-3) (2000) 15-22. doi: 10.1103/PhysRevLett.97.131801.

[8] A. Serebrov, et al., Phys. Rev. C 78 (3) (2008) 035505-035520. doi : 10.1103/PhysRevC.78.035505.

[9] M. Mendenhall, et al., Phys. Rev. C 87 (3) (2013) 032501-032507. doi : 10.1103/PhysRevC.87.032501.

[10] A. Saunders, et al., Rev. Sci. Instrum. 84 (1) (2013) 013304. doi:10. 1063/1.4770063.

[11] B. Lauss, Physics Procedia 51 (2014) 98-101. doi : $10.1016 /$ j.phpro. 2013.12 .022$.

[12] Y. Masuda, et al., Phys. Rev. Lett. 108 (13) (2012) 134801. doi:10. 1103/PhysRevLett.108.134801.

[13] E. Korobkina, et al., Physics Letters A 301 (2002) 462.

[14] J. Karch, et al., Eur. Phys. J. A 50 (4) (2014) 78. doi:10.1140/epja/ i2014-14078-9.

[15] R. Golub, J. Pendlebury, Phys. Lett. A 53 (2) (1975) 133-135. doi: $10.1016 / 0375-9601(75) 90500-9$.

[16] C. Morris, et al., Phys. Rev. Lett. 89 (27) (2002) 272501. doi:10.1103/ PhysRevLett.89.272501.

[17] R. B. Scott, et al., J. Chem. Phys 454 (1934). doi : 10.1063/1.1749509.

[18] C.-Y. Liu, et al., Nucl. Instr. Meth. A 508 (257). doi:10.1016/ S0168-9002(03) 01666-8.

[19] F. Atchison, et al., J. Res. Natl. Inst. Stand. Technol. 110 (4) (2005) 491. doi:10.6028/jres.110.076

[20] K. Bodek, et al., Nucl. Instr. Meth. A 533 (3) (2004) 491-504. doi: 10.1016/j.nima.2004.06.157.

[21] N. Sullivan, et al., Cryogenics 30 (8) (1990) 734-735. doi:10.1016/ 0011-2275 (90) 90240-D.

[22] H. Kuhn, et al., Principles of Physical Chemistry, 2nd Edition, Wiley, 2000 .

[23] RSICC MCNP6 Monte Carlo N-Particle Transport Code, version 1.0 (2013). [link].

URL http://mcnp.lanl.gov 
437 [24] E. Gutsmiedl, et al., Nucl. Instr. Meth. A 611 (2-3) (2009) 256-258. doi :

$438 \quad 10.1016 / j . n i m a .2009 .07 .082$.

439 [25] A. Mueller, PhD thesis, TU Muenchen (2008). 